\title{
A novel CYP7A1 polymorphism is associated with the low-density lipoprotein cholesterol response to atorvastatin
}

\author{
Zhi-Ke Liu', Wotan Zeng ${ }^{2}$, Yan Zhang ${ }^{3}$, Jia Jia ${ }^{3}$, Jian-Ping $\mathrm{Li}^{3}$, Yong Huo ${ }^{3}$ and Da-Fang Chen ${ }^{1 *}$ \\ ${ }^{1}$ Department of Epidemiology and Biostatistics, School of Public Health, Peking University Health Science Center, Beijing 100191, China \\ ${ }^{2}$ East Asian Gene Science and Technology Research Institute, Beijing, 100078, China \\ ${ }^{3}$ Department of Cardiology, Peking University First Hospital, Beijing 100034, China
}

\begin{abstract}
Backgrounds and aims: Cholesterol $7 \alpha$ hydroxylase encoding by gene CYP7A1 is the initial and rate-limiting step in the classical bile acid synthesis pathway. Atorvastatin can markedly upregulate the mRNAs of bile acids synthetic enzymes CYP7A1 in the liver to increase fecal bile acid excretion. We tempt to investigate the association between a novel CYP7A1 polymorphism rs8192875 and reduction of lipid levels response to atorvastatin in Chinese patients with coronary artery disease.

Methods: Of 169 patients with coronary artery disease were treated with atorvastatin for one month. Lipid profiles, including triglycerides(TGs), total cholesterol(TC), low-density lipoprotein cholesterol(LDL-C), and high-density lipoprotein cholesterol(HDL-C) were determined before and after treatment. Rs8192875 genotypes were assayed with the iPLEX assay in conjunction with the MassARRAY platform. We performed independent sample $t$ test or Kruskal-Wallis test to evaluate the effects of SNP.

Results: After one month of atorvastatin therapy, the lipid levels decreased significantly. Compared with AG genotype, the GG genotype of rs8192875 achieved a greater reduction of LDL-C level $(0.694 \pm 0.701$ vs. $0.136 \pm 0.401 \mathrm{mmol} / 1, p=0.0056 ; 24.090 \pm 23.104 \%$ vs. $2.182 \pm 20.809 \%, p=0.0031)$; and a similar pattern of efficacy appears to TC $(0.808 \pm 0.791$ vs. $0.302 \pm 0.381 \mathrm{mmol} / 1, p=0.0208 ; 16.410 \pm 15.370 \%$ vs. $6.936 \pm 9.711 \%$, $p=0.0341)$. The genotypes had no significant difference on TGs or HDL cholesterol-lowering response to atorvastatin.
\end{abstract}

Conclusions: A novel CYP7A1 exon variant rs8192875 is significantly associated with reducing LDL-C and TC level response to atorvastatin.

\section{Introduction}

Statins are most widely prescribed to lowering circulating concentrations of LDL cholesterol for the prevention of CVD [1]. Several meta-analyses from randomized clinical trials have manifested that a statin-mediated reduction of $1 \mathrm{mmol} / \mathrm{l}$ in $\mathrm{LDL}$ cholesterol yield a proportional reduction of approximately $20 \%$ in major vascular events $[2,3]$. And in general, the greater reductions in LDL cholesterol level, the larger reductions in vascular disease risk.

Most of the patients are extremely well tolerated to the treatment of statins, but some others have intolerable muscle adverse drug reactions with symptoms ranging from mild myalgia to fatal rhabdomyolysis [4]. The risk tends to increase with the increasing dosage of statins. Such as myalgia, the reported incidence is $3-5 \%$ in clinical trials [5] and up to $10 \%$ among the patients of high-dose statins [6]. In addition, statin therapy has been insufficient in some patients, $40 \%$ of high-risk and $80 \%$ of very-high-risk individuals do not meet their LDL cholesterol targets [7]. Moreover, it is estimated that genetic determinants account for $20-95 \%$ of the variability in drug disposition and effects $[8,9]$. In the classical bile acid synthesis pathway, cholesterol $7 \alpha$ hydroxylase encoding by gene CYP7A1 is the initial and rate-limiting step for the removal of cholesterol into bile [10]. And then atorvastatin can markedly upregulate, covering 10-fold, the mRNAs of the bile acids synthetic enzymes CYP7A1 in the liver to increase fecal bile acid excretion [11]. Therefore, the gene CYP7A1 was chosen as a major candidate gene to investigate the individual sensitivity response to atorvastatin. At present, several studies have reported that CYP7A1 polymorphisms influence the LDL cholesterol-lowering response to atorvastatin [1215], such as the CYP7A1 A-204C*A in Caucasians and rs8192870*T in Chineses have a greater reduction in LDL cholesterol level response to atorvastatin as compared with variant allele-carrying genotype. Above information have urged us to research optimal atorvastatin treatment options, which aims at maximizing reduction of LDL cholesterol at the risk of minimal adverse effects. In this study, we preliminarily tempt to investigate the association between a novel CYP7A1 polymorphism rs8192875 and reductions of lipid levels response to atorvastatin in Chinese patients with coronary artery disease.

\section{Materials and methods}

\section{Subjects}

This project was designed as a hospital-based prospective study and conducted by Peking University First Hospital (Beijing, China). Patients are eligible for the study who are scheduled for elective coronary

Correspondence to: Chen Da-fang, Department of Epidemiology and Biostatistics, Peking University Health Science Center, 38 Xueyuan Rd., Haidian District, Beijing 100191, P.R. China, Tel:+ 86-10-82802644; Fax: +86-1082802644; E-mail: dafangchen@bjmu.edu.cn

Key words: pharmacogenetics; CYP7A1; atovarstatin; lipid-lowing

Received: June 17, 2017; Accepted: July 07, 2017; Published: July 10, 2017 
angiography and who have been clinically diagnosed with either NSTEACS (unstable angina or NSTE acute myocardial infarction) or stable angina pectoris. Patients with following conditions were excluded from the study: have LDL-C $<1.8 \mathrm{mmol} / \mathrm{L}$ without statin therapy within 1 month; have uncontrolled clinically significant disease; have active hepatic disease or hepatic dysfunction are allergic or have experienced serious adverse reaction to HMG-CoA reductase; are participating in other interventional clinical trials using drugs or devices.

Between January 2010 and December 2011, a total of 165 consecutive unrelated adult patients in 20-75 years old with coronary artery disease were enrolled into the study. Of these subjects, 104 patients receive intensive atorvastatin therapy (atorvastatin $80 \mathrm{mg} /$ day for two consecutive days before and $40 \mathrm{mg} /$ day for 28 days after percutaneous coronary intervention); 65 patients receive routine atorvastatin therapy (less than atorvastatin $20 \mathrm{mg}$ /day daily for 30 days). The study was approved by the Ethics Committee of the Peking University Health Science Center, and all the subjects delivered written informed consent for participation.

In the first visit, demographic, behavioral information, clinical characteristics were collected from trained physicians, including age, gender, height, weight, smoking and drinking habits and medical history information. After one month, patients come back for a clinical follow-up. A clinical follow-up questionnaire was finished by a trained physician for monitoring the subjects' medication compliance and side effects.

\section{Lipids tests}

Over 8 hours fasting blood samples before and after one month of treatment with atorvastatin were collected for the measurement of lipid profiles. All biochemistry tests were performed by uniformly standard methods in the clinical laboratory of the Peking university First Hospital via an automatic biochemical analyzer.

\section{DNA preparation and genotyping}

Genomic DNA was extracted from the participants' peripheral blood leucocytes by using the protein precipitation method following standard procedures. Each DNA sample was quantified using a Nanophotometer (Implen, Germany) and was diluted to a final concentration of $50 \mathrm{ng} / \mathrm{uL}$.

The rs8192875 genotypes were assayed with the iPLEX assay in conjunction with the MassARRAY platform (Sequenom, La Jolla, CA) according to the manufacturer's protocol. The SNP had a call rate of greater than $95 \%, 5 \%$ of the blind duplicate samples were genotyped repeatedly and the rate of consistency was $100 \%$.

\section{Statistical analysis}

All statistical analyses were performed using SAS9.2 (SAS institute Inc., Cary, NC). A logarithmic transformation was applied to skewed distribution data before analysis. Means \pm standard deviation (SD) are given for all continuous variables and absolute numbers and percentages for category variables. The $\chi 2$ test was used to examine the Hardy-Weinberg equilibrium. Differences between lipid parameters at baseline and after 30 days treatment were tested using paired-t-test. Category variables differences among the genotypes were analyzed using Pearson $\chi 2$ tests or Fisher's exact test of probabilities. The absolute reductions and relative changes of plasma lipid parameter levels among genotypes were tested using independent sample t-test or Kruskal-Wallis test. Two-sided tests were adopted and a p-value of $<0.05$ was considered statistically significant.

\section{Results}

\section{Genotyping}

A total of 169 patients with good adherence to atorvastatin treatment were included in the study. The genotypes were consistent with the Hardy-Weinberg equilibrium $(\chi 2=0.01, p=0.935)$. Frequencies of the $\mathrm{G}$ and $\mathrm{A}$ alleles were 0.973 and 0.027 , respectively. The variant allele was basically same, as compared to the population HAPMAPCHB (0.049) ( $\mathrm{p}=0.198$ by Pearson $\chi^{2}$ test).

\section{Baseline characteristics of participants}

The mean age of the study subjects was $59.34 \pm 8.16$ years and $72.8 \%$ were male $(n=123)$. The baseline characteristics of the study participants are shown in Table1. There were no significant differences in baseline demographic characteristics or clinical disease history between the rs8192875 genotype groups (Table1).

\section{Lipid responses}

Treatment with atorvastatin therapy daily for one month significantly reduced TC from $1.443 \pm 0.219$ to $1.264 \pm 0.210 \mathrm{mmol} / \mathrm{L}$ $(p<0.001), \mathrm{LDL}-\mathrm{C}$ from $0.913 \pm 0.317$ to $0.610 \pm 0.335 \mathrm{mmol} / \mathrm{L}$ $(p<0.001)$ and TG from $0.441 \pm 0.478$ to $0.190 \pm 0.404 \mathrm{mmol} / \mathrm{L}$ $(p<0.001)$, while increased HDL-C from $0.027 \pm 0.239$ to $0.088 \pm 0.271$ $\mathrm{mmol} / \mathrm{L}(p=0.006)$ (Table 2).

\section{Effect of the genetic polymorphisms on the lipids responses to atorvastatin}

There was no statistically significant difference in all baseline lipid profiles, while significant differences in the absolute reduction and percentage change in TC and LDL-C level between rs8192875 genotype groups after one month of atorvastatin therapy. Patients with genotype GG/rs8192875 had a $21.9 \%(0.558 \mathrm{mmol} / \mathrm{l})$ greater reduction in LDL-C level than those with genotype AG/rs8192875 (0.694 \pm 0.701 vs. 0.136 $\pm 0.401 \mathrm{mmol} / \mathrm{l}, p=0.0056 ; 24.090 \pm 23.104 \%$ vs. $2.182 \pm 20.809 \%$, $p=0.0031)$. And the changes in TC appeared a similar pattern, a $9.47 \%$ or $0.506 \mathrm{mmol} / \mathrm{l}$ greater reduction in TC in genotype GG/rs 8192875 subjects as compared with genotype AG/rs8192875 subjects $(0.808 \pm$ 0.791 vs. $0.302 \pm 0.381 \mathrm{mmol} / \mathrm{l}, p=0.0208 ; 16.410 \pm 15.370 \%$ vs. $6.936 \pm$ $9.711 \%, p=0.0341$ ). The genotypes had no significant effects on TG or HDL-C response to atorvastatin (Table 3 ).

Table 1. Baseline demographic and clinical characteristics of patients between CYP7A1 rs8192875.

\begin{tabular}{|c|c|c|c|c|}
\hline \multirow{2}{*}{ Variables $\dagger$} & \multicolumn{4}{|c}{ CYP7A1 rs8192875 } \\
\hline $\begin{array}{c}\text { Intensive } \\
\text { atorvastin }\end{array}$ & $106(62.7)$ & $102(63.8)$ & $4(44.4)$ & 0.244 \\
\hline Age(years) & $59.34 \pm 8.16$ & $59.46 \pm 8.21$ & $57.22 \pm 7.19$ & 0.318 \\
\hline Male & $123(72.8)$ & $118(73.8)$ & $5(55.6)$ & 0.233 \\
\hline BMI(kg/m $\left.{ }^{2}\right)$ & $25.34 \pm 3.16$ & $25.30 \pm 3.23$ & $26.03 \pm 1.30$ & 0.166 \\
\hline Current Smoker & $69(40.8)$ & $67(41.9)$ & $2(22.2)$ & 0.1505 \\
\hline Chronic Statin & $64(37.9)$ & $60(37.5)$ & $4(44.4)$ & 0.676 \\
\hline Hypertension & $104(61.5)$ & $100(62.5)$ & $4(44.4)$ & 0.279 \\
\hline T2DM & $43(25.4)$ & $41(25.6)$ & $2(22.2)$ & 0.3055 \\
\hline Stroke & $14(8.3)$ & $12(7.5)$ & $2(22.2)$ & 0.1356 \\
\hline PVD & $9(5.3)$ & $8(5.0)$ & $1(11.1)$ & 0.3216 \\
\hline
\end{tabular}

$\uparrow$ : Continuous variables were presented as mean \pm standard deviation, and categorical variables were presented as n (\%); BMI: body mass index; T2DM: type 2 diabetes mellitus; PVD: Peripheral Vascular Diseases. 
Table 2. Comparison of Plasma lipid parameters before and after treatment with atorvastatin.

\begin{tabular}{|c|c|c|c|}
\hline Variables $\dagger$ & Baseline & 30 Days & $\boldsymbol{P}$ \\
\hline $\mathrm{TC}(\mathrm{mmol} / \mathrm{l})$ & $1.443 \pm 0.219$ & $1.264 \pm 0.210$ & $<0.001$ \\
\hline $\mathrm{LDL}-\mathrm{C}(\mathrm{mmol} / \mathrm{l})$ & $0.913 \pm 0.317$ & $0.610 \pm 0.335$ & $<0.001$ \\
\hline $\mathrm{TG}(\mathrm{mmol} / \mathrm{l})$ & $0.441 \pm 0.478$ & $0.190 \pm 0.404$ & $<0.001$ \\
\hline HDL-C $(\mathrm{mmol} / \mathrm{l})$ & $0.027 \pm 0.239$ & $0.088 \pm 0.271$ & 0.006 \\
\hline
\end{tabular}

$\dagger$ : variables were presented as mean \pm standard deviation; $P$ :analysed by paired $\mathrm{t}$ test.

Table 3. The association of rs 8192875 with lipid lowering effects reponse to atorvastatin.

\begin{tabular}{|c|c|c|c|}
\hline \multirow{2}{*}{ Variables $\dagger$} & \multicolumn{2}{|c|}{ CYP7A1 rs8192875 } & P \\
\hline TC & GG (N=162) & AG (N=9) & \\
\hline Baseline(mmol/l) & $1.448 \pm 0.219$ & $1.367 \pm 0.209$ & $0.2838^{*}$ \\
\hline Reduction (abs) & $0.808 \pm 0.791$ & $0.302 \pm 0.381$ & 0.0208 \\
\hline Reduction (per) & $16.410 \pm 15.370$ & $6.936 \pm 9.711$ & 0.0341 \\
\hline LDL-C & & & \\
\hline Baseline(mmol/1) & $0.919 \pm 0.318$ & $0.816 \pm 0.300$ & $0.3416^{*}$ \\
\hline Reduction (abs) & $0.694 \pm 0.701$ & $0.136 \pm 0.401$ & 0.0056 \\
\hline Reduction (per) & $24.090 \pm 23.104$ & $2.182 \pm 20.809$ & 0.0031 \\
\hline TG & & & \\
\hline Baseline(mmol/l) & $0.436 \pm 0.480$ & $0.543 \pm 0.457$ & $0.5391 *$ \\
\hline Reduction (abs) & $0.414 \pm 0.580$ & $0.523 \pm 0.811$ & 0.9189 \\
\hline Reduction (per) & $17.648 \pm 25.870$ & $17.417 \pm 24.669$ & 0.6009 \\
\hline HDL-C & & & \\
\hline Baseline(mmol/1) & $0.029 \pm 0.237$ & $-0.006 \pm 0.28$ & 0.4752 \\
\hline Reduction (abs) & $-0.058 \pm 0.197$ & $-0.004 \pm 0.233$ & 0.3207 \\
\hline Reduction (per) & $-5.512 \pm 18.889$ & $-3.416 \pm 22.208$ & 0.5793 \\
\hline
\end{tabular}

$\dagger$ : variables were presented as mean \pm standard deviation; $P^{*}$ : analysed by $\mathrm{T}$ test for two independent sample; $P$ :analysed by Kruskal-Wallis Test.

\section{Discussion}

In the present study, the association of CYP7A1 rs8192875 polymorphism with lipid lowering effect respond to atorvastatin is statistically significant. There is a greater reduction in LDL cholesterol level and Total cholesterol level in subjects with genotype GG in contrast to the subjects with genotype AG, $21.9 \%(0.558 \mathrm{mmol} / \mathrm{l})$ and $9.47 \%(0.506 \mathrm{mmol} / \mathrm{l})$ respectively.

Previous pharmacogenetics studies aiming at evaluating the potential contribution of CYP7A1 polymorphisms to the observed variability in response to atorvastatin therapy have accumulated evidence of a causal relationship between them. In Caucasians, SNP rs3808607 (A-240C) has been reported to be associated with plasma LDL cholesterol level [16] and LDL cholesterol lowering reduction in response to atorvastatin [12,13]. Besides, three-loci interaction comprising of CYP7A1(rs8192871AA)/APOAI(PstP1P1)/HMGCR (rs12916CT) was found to be associated with a maximum decrease in LDL cholesterol levels. Above associations about SNP rs3808607 are either inconsistent [14] or unobserved [15], but Wei et al. found the CYP7A1 -204A and ABCG8 1199A alleles appear to interact to affect lipid-lowering response to atorvastatin [14]. In addition, Jiang et al. applied a gene-wide tagging SNP strategy to study all common variants of CYP7A1 (minor allele frequency > 5\%) on atorvastatin response, and the results show that genotype TT/rs8192870, in the first intron of CYP7A1 gene, had greater reduction of LDL cholesterol in response to atorvastatin [15]. Here, we report for the first time, that SNP rs8192875 is significantly associated with LDL cholesterol and total cholesterol-lowering response to atorvastatin. The National Center for Biotechnology Information (NCBI) annotate that SNP rs8192875 is a functional polymorphism located in the exon of CYP7A1, and its heterozygote or homozygote variant leads to missense mutation
(Asp347Asn) [17], so we can assume that the variant may result in the decrease of the active site and enzyme function.

Cholesterol $7 a$ hydroxylase (CYP7A1), which catalyzes the hydroxylation of cholesterol at the $7 \alpha$ position in the liver, the first reaction in the classic pathway of bile acid synthesis, and that represents the check-point of the whole process. Bile acid metabolism is strongly related to whole-body cholesterol homeostasis; bile acid synthesis and bile acid-facilitated biliary cholesterol secretion are the only significant pathways for cholesterol excretion from the body [18]. And in general, in humans, the major fraction of cholesterol is transported associated to LDL particles [19]. In 2002, Pullinger et al. elaborated the first and so far, only reported hypercholesterolemic phenotype of CYP7A1 deficiency in humans caused by frameshift mutations (L413fsX414) in exon 6 [20]. This information suggests that CYP7A1 enzyme activity profoundly influences cholesterol homeostasis.

There were some limitations in this study. First, the sample size of the study is small, the statistical power is not enough for further detecting the effect of interaction or analyzing the subgroup differences. A more large prospective study is warranted. Second, we did not test the level of lipoprotein(a). Lipoprotein(a) account for approximately $45 \%$ cholesterol, the lipoprotein(a) level may have a direct effect on the LDL cholesterol response to atorvastatin [21]. Third, compared with previous evidence, the one-month duration of the study was not long enough to observe a long-term effect of SNP rs8192875 on the efficacy of atorvastatin therapy. Despite the limitations mentioned above, our study has several strengths. The monotherapy of atorvastatin could avoid the concomitant medications influence. And prospective design could contribute to obtaining the information without recall bias. Moreover, the credibility of the associations of this research was enhanced by the similar pattern of lowering efficacy in LDL cholesterol and total cholesterol between genotypes of SNP rs8192875 response to atorvastatin. Above all, further functional research, as well as pharmacogenomic studies with good design and longer duration among other ethnic groups, is warranted.

\section{Conclusion}

The data presented here demonstrate that a CYP7A1 exon variant rs8192875 is significantly associated with response to atorvastatin in terms of LDL cholesterol and total cholesterol lowering. Patients with GG genotype yield a greater response to atorvastatin in terms of the reduction of LDL cholesterol and total cholesterol than those with AG genotype. These findings offer a new potential way to optimize the clinical therapy for individual patients in need of lipid-lowering.

\section{Conflicts of interest}

None of the authors has a conflict of interests with regard to the publication of this study.

\section{Funding}

The study was supported by a grant from the UM-PUHSC joint institute for translational and clinical research (BMU20110177). The authors have no conflicts of interest to declare.

\section{References}

1. Shepherd J, Hunninghake DB, Barter P, McKenney JM, Hutchinson HG (2003) Guidelines for lowering lipids to reduce coronary artery disease risk: a comparison of rosuvastatin with atorvastatin, pravastatin, and simvastatin for achieving lipid-lowering goals. Am J Cardiol 91: 11C-17C. [Crossref]

2. Baigent C, Keech A, Kearney PM, Blackwell L, Buck G, et al. (2005) Efficacy and safety of cholesterol-lowering treatment: prospective meta-analysis of data from 90,056 participants in 14 randomised trials of statins. Lancet 366: 1267-1278. [Crossref] 
3. Mihaylova B, Emberson J, Blackwell L, Keech A, Simes J, et al. (2012) The effects of lowering LDL cholesterol with statin therapy in people at low risk of vascular disease: meta-analysis of individual data from 27 randomised trials. Lancet 380: 581-590. [Crossref]

4. Thompson PD, Clarkson PM, Rosenson RS (2006) National lipid association statin safety task force muscle safety expert panel (2006) an assessment of statin safety by muscle experts. Am J Cardiol 97: 69C-76C. [Crossref]

5. Thompson PD, Clarkson P, Karas RH (2003) Statin-associated myopathy. JAMA 289: 1681-1690. [Crossref]

6. Bruckert E, Hayem G, Dejager S, Yau C, Begaud B (2005) Mild to moderate muscular symptoms with high-dosage statin therapy in hyperlipidemic patients--the PRIMO study. Cardiovasc Drugs Ther 19: 403-414.

7. Yan AT, Yan RT, Tan M, Hackam DG, Leblanc KL, et al. (2006) Contemporary management of dyslipidemia in high-risk patients: targets still not met. Am J Med 119: 676-683. [Crossref]

8. Weinshilboum R (2003) Inheritance and drug response. N Engl J Med 348: 529-537. [Crossref]

9. Evans WE, McLeod HL (2003) Pharmacogenomics--drug disposition, drug targets, and side effects. $N$ Engl J Med 348: 538-549. [Crossref]

10. Chiang JY (2009) Bile acids: regulation of synthesis. J Lipid Res 50: 1955-1966. [Crossref]

11. Fu ZD, Cui JY, Klaassen CD (2014) Atorvastatin induces bile acid-synthetic enzyme Cyp7a1 by suppressing FXR signaling in both liver and intestine in mice. $J$ Lipid Res 55: 2576-2586. [Crossref]

12. Kajinami K, Brousseau ME, Ordovas JM, Schaefer EJ (2005) A promoter polymorphism in cholesterol 7alpha-hydroxylase interacts with apolipoprotein E genotype in the LDLlowering response to atorvastatin. Atherosclerosis 180: 407-415. [Crossref]
13. Poduri A, Khullar M, Bahl A, Sehrawat BS, Sharma Y, Talwar KK (2010) Common variants of HMGCR, CETP, APOAI, ABCB1, CYP3A4, and CYP7A1 genes as predictors of lipid-lowering response to atorvastatin therapy. DNA Cell Biol 29: 629637. [Crossref]

14. Wei KK, Zhang LR, Zhang Y, Hu XJ (2011) Interactions between CYP7A1 A-204C and ABCG8 C1199A polymorphisms on lipid lowering with atorvastatin. J Clin Pharm Ther 36: 725-733. [Crossref]

15. Jiang XY, Zhang Q, Chen P, Li SY, Zhang NN, et al. (2012) CYP7A1 polymorphism influences the LDL cholesterol-lowering response to atorvastatin. J Clin Pharm Ther 37: 719-723. [Crossref]

16. Couture P, Otvos JD, Cupples LA, Wilson PW, Schaefer EJ, et al. (1999) Association of the A-204C polymorphism in the cholesterol 7alpha-hydroxylase gene with variations in plasma low density lipoprotein cholesterol levels in the Framingham Offspring Study. J Lipid Res 40: 1883-1889. [Crossref]

17. http://www.ncbi.nlm.nih.gov/projects/SNP/snp_ref.cgi?rs=8192875.

18. Li T, Francl JM, Boehme S, Chiang JY (2013) Regulation of cholesterol and bile acid homeostasis by the cholesterol 7alpha-hydroxylase/steroid response element-binding protein 2/microRNA-33a axis in mice. Hepatology 58: 1111-1121. [Crossref]

19. Gilardi F, Mitro N, Godio C, Scotti E, Caruso D, et al. (2007) The pharmacological exploitation of cholesterol 7alpha-hydroxylase, the key enzyme in bile acid synthesis from binding resins to chromatin remodelling to reduce plasma cholesterol. Pharmacol Ther 116: 449-472. [Crossref]

20. Pullinger CR, Eng C, Salen G, Shefer S, Batta AK, et al. (2002) Human cholestero 7alpha-hydroxylase (CYP7A1) deficiency has a hypercholesterolemic phenotype. $J$ Clin Invest 110: 109-117. [Crossref]

21. Miltiadous G, Saougos V, Cariolou M, Elisaf MS (2006) Plasma lipoprotein(a) levels and LDL-cholesterol lowering response to statin therapy in patients with heterozygous familial hypercholesterolemia. Ann Clin Lab Sci 36: 353-355. [Crossref]

Copyright: (C2017 Liu Z. This is an open-access article distributed under the terms of the Creative Commons Attribution License, which permits unrestricted use, distribution, and reproduction in any medium, provided the original author and source are credited. 